\title{
ON BASES IN THE DISC ALGEBRA
}

\author{
BY
}

\author{
J. BOURGAIN
}

\begin{abstract}
It is shown that the disc algebra has no Besselian basis. In fact, concrete minorations on certain Lebesgue functions are obtained. A consequence is the nonisomorphism of the disc algebra and the space of uniformly convergent Fourier series on the circle.
\end{abstract}

0. Introduction. The purpose of this paper is to prove the following result on the disc algebra $A$.

THEOREM. Assume $m$ is a positive integer and $\left(\varphi_{k}\right)_{1 \leq k \leq n},\left(x_{k}\right)_{1 \leq k \leq n}$ are biorthogonal sequences in $A, A^{*}$ resp., satisfying the properties:

1. $\left\|\varphi_{k}\right\|_{\infty} \leq M(1 \leq k \leq n)$,

2. $\left\|\sum a_{k} x_{k}\right\| \leq M\left(\sum\left|a_{k}\right|^{2}\right)^{1 / 2}$ for all scalar sequences $\left(a_{k}\right)$. Then, for some $z \in \mathbf{C},|z| \leq 1$,

$$
\frac{1}{n} \sum_{1 \leq m \leq n}\left\|\sum_{1 \leq k \leq m} \varphi_{k}(z) x_{k}\right\| \geq \rho(M) \log n .
$$

This theorem can be seen as an extension to the disc algebra of results of A. M. Olevskii [9] and S. V. Bochkarev [5]. The next consequence is immediate. Its analogue for the spaces $C$ and $L^{1}$ was proved by S. Szarek (see [11], also for definitions).

COROllary 1. The space $A$ (resp. $L^{1} / H_{0}^{1}$ ) has no Besselian (resp. Hilbertian) basis.

Recall that a sequence $\left(x_{k}\right)_{k=1,2, \ldots}$ is called Besselian (resp. Hilbertian) provided it admits a lower (resp. upper) estimation

$$
\left\|\sum a_{k} x_{k}\right\| \geq c\left(\sum\left|a_{k}\right|^{2}\right)^{1 / 2} \quad\left(\text { resp. }\left\|\sum a_{k} x_{k}\right\| \leq C\left(\sum\left|a_{k}\right|^{2}\right)^{1 / 2}\right) .
$$

Notice that the space $A$ (resp. $L^{1} / H^{1}$ ) has a basis (see $[6]$ ).

The above theorem permits a local version of Corollary 1 in terms of finitedimensional complemented subspaces of these spaces. Denote by $U$ the completion of the space of analytic polynomials on the circle (or on the disc) under the norm

$$
\|p\|_{U}=\sup _{m}\left\|p * D_{m}\right\|_{\infty}
$$

where $D_{m}(\theta)=\sum_{0 \leq k \leq m} e^{i k \theta}$ is the corresponding Dirichlet kernel.

Since obviously the characters form a Besselian basis for $U$, another consequence is $($ see $[\boldsymbol{3}])$.

Received by the editors January 22, 1983 and, in revised form, September 13, 1983.

1980 Mathematics Subject Classification. Primary 46E15, 46B15. 
COROLlaRY 2. There is no linear isomorphism between the spaces $A$ and $U$.

We use the notation $C$ for a numerical constant. In what follows, elements of $A$ and $H^{\infty}$ will be considered as functions on the circle $\Pi$.

The reader may consult [8] as standard reference for general Banach space theory and $[\mathbf{1 0}]$ for Banach spaces of analytic functions.

1. Lifting and change of measure. By an argument of local reflexivity (see $\left[8\right.$, p. 33] for details), it is clear that we can assume $x_{k} \in L^{1} / H_{0}^{1}$. Consider the operator from $A$ into $l_{n}^{2}$ mapping $\varphi$ onto the sequence $\left(\left\langle\varphi, x_{k}\right\rangle\right)_{1 \leq k \leq n}$. The norm of this operator is bounded by $M$ and by the result of [1] (see $\S 2$ ) has 2-summing norm at most $C M$. Hence, using the Pietch-Grothendieck factorization theorem (cf. $[11$, p. 58]) and a change of density, we get

LEMMA 1. There are functions $\left(f_{k}\right)_{1 \leq k \leq n}$ in $L^{1}(\Pi)$ and $\Delta \in L^{1}(\Pi), \Delta>0$ and $\int \Delta=1$ satisfying:

(i) $q\left(f_{k}\right)=x_{k}(1 \leq k \leq n)$ where $q$ denotes the quotient map from $L^{1}(\Pi)$ onto $L^{1} / H_{0}^{1}$,

(ii) $\left(\int|f|^{2} \Delta^{-1}\right)^{1 / 2} \leq C M\left(\sum\left|a_{k}\right|^{2}\right)^{1 / 2}$ for each $f=\sum a_{k} f_{k}$

Note that indeed, since $x_{k} \in L^{1} / H_{0}^{1}$, we can assume that the corresponding Pietch measure is of the form $\Delta d m$ ( $m$ is the Haar measure on $\Pi$ ).

To $\Delta$ we apply Proposition 1.2 of $[\mathbf{2}]$, which we restate now.

LEMMA 2. There are positive scalars $\left(c_{i}\right)$ and $H^{\infty}$-functions $\left(\theta_{i}\right),\left(\tau_{i}\right)$ such that defining $\Delta_{1}=\sum c_{i}\left|\tau_{i}\right|$ :

(i) $\left\|\theta_{i}\right\|_{\infty} \leq 1$,

(ii) $\left\|\sum\left|\tau_{i}\right|\right\|_{\infty} \leq C$,

(iii) $\sum \tau_{i}^{2} \theta_{i}=1$ a.e.,

(iv) $\Delta_{1} \geq \max (1, \Delta)$ and $\int \Delta_{1} \leq C$,

(v) $\left|\tau_{i}\right| \Delta_{1} \leq C c_{i}$ a.e.

It will be convenient to replace the Haar measure $m$ on $\Pi$ by the measure $d \mu=$ $\Delta_{1} d m$.

The following fact will be exploited in the sequel.

Lemma 3. Assume $\alpha \in L^{2}(\mu), \alpha \geq 0$. Then there exists $\psi \in H^{\infty}$ so that:

(i) $\|\psi\|_{\infty} \leq C$,

(ii) $|\psi| \cdot \alpha \leq 1$ a.e.,

(iii) $\|1-\psi\|_{L^{2}(\mu)} \leq C\|\alpha\|_{L^{2}(\mu)}$.

PROOF. It is clearly sufficient to estimate in (ii) by some constant $c$. Using the notations of Lemma 2, consider for each $i$ the function $\gamma_{i}=\left(1+\left|\tau_{i}\right| \alpha\right)^{-1}$ and the outer functors $\psi_{i}$ with boundary value $\gamma_{i} \exp \left[i \mathcal{H}\left(\log \gamma_{i}\right)\right]$ ( $H=$ Hilbert-transform). Then

$$
\left\|1-\psi_{i}\right\|_{2} \leq C\left\|\left|\tau_{i}\right| \cdot \alpha\right\|_{2} \quad \text { and } \quad \alpha\left|\tau_{i}\right| \cdot\left|\psi_{i}\right| \leq 1 \quad \text { a.e. }
$$


Define $\psi=\sum \theta_{i} \tau_{i}^{2} \psi_{i}$. Then, by (ii) of Lemma $2,\|\psi\|_{\infty} \leq 1$ and $\alpha \cdot|\psi| \leq c$ a.e. By (iii) and (v) of Lemma 2

$$
\begin{aligned}
\int|1-\psi|^{2} \Delta_{1} & \leq C \int \sum\left|\tau_{i}\right|^{2}\left|1-\psi_{i}\right|^{2} \Delta_{1} \leq C \sum c_{i}\left\|1-\psi_{i}\right\|_{2}^{2} \\
& \leq C \int \alpha^{2}\left(\sum c_{i}\left|\tau_{i}\right|^{2}\right) \leq C \int \alpha^{2} \Delta_{1},
\end{aligned}
$$

which completes the proof.

Let us replace $f_{k}$ by $\bar{f}_{k}=f_{k} \Delta_{1}^{-1}$ for each $k=1, \ldots, n$. Define further for $f \in L^{1}(\mu)$

$$
\|f\|_{*}=\sup _{\substack{\varphi \in H^{\infty} \\\|\varphi\|_{\infty} \leq 1}}\left|\int f \cdot \varphi d \mu\right| .
$$

Hence $\|f\|_{L^{2}(\mu)} \leq C M\left(\sum\left|a_{k}\right|^{2}\right)^{1 / 2}$ for $f=\sum a_{k} \bar{f}_{k}$ and the inequality stated in the theorem becomes

$$
\frac{1}{n} \sum_{1 \leq m \leq n}\left\|\sum_{1 \leq k \leq m} \varphi_{k}(z) \bar{f}_{k}\right\|_{*} \geq \rho(M) \log n .
$$

2. Expression of the mean using Haar and Schauder systems. It is no restriction to take $n$ of the form $n=2^{R}$, thus $R=\log n$. Denote by $\left(\chi_{j}(\omega)\right)_{j}$ the $L^{\infty}$-normalized Haar system on $[0,1]$ and $\left(\zeta_{j}(s)\right)_{j}$ the Schauder system on $[0,1]$ (see $[9$, p. 36]). We reformulate the left member in $(*)$ as in $[5]$. Define the function $F$ on $\Pi \times \Pi \times[0,1]$ by

$$
F(\theta, \psi, s)=\varphi_{k}(\theta) \bar{f}_{k}(\psi) \quad \text { if }(k-1) / n \leq s<k / n
$$

and for $m=1,2, \ldots, n$

$$
\Lambda_{m}(s)= \begin{cases}1 & \text { if } s \in[0, m / n] \\ 0 & \text { otherwise }\end{cases}
$$

Notice that for fixed $\theta$ and $\zeta \in L^{2}[0,1]$

$$
\left\|\int F(\theta, \psi, s)_{\zeta}(s) d s\right\|_{L^{2}(\mu(d \psi))} \leq C M^{2} n^{-1 / 2}\left\|_{\zeta}\right\|_{2} .
$$

For $z=e^{i \theta}$, the left member of $(*)$ becomes

$$
\sum_{1 \leq m \leq n}\left\|\int F(\theta, \psi, s) \Lambda_{m}(s) d s\right\|_{*} .
$$

For $\omega \in\left[(m-1) n^{-1}, m n^{-1}\right]$, one has (see $[7$, pp. 120, 50])

$$
\Lambda_{m}(s)=1-s-\frac{1}{2} \sum_{r=0}^{R-1} \sum_{j=2^{r}+1}^{2^{r+1}} \chi_{j}(\omega) \zeta_{j}(s)+\lambda(\omega, s),
$$


where $\lambda(\omega, s)$ is supported by $\left[(m-1) n^{-1}, m n^{-1}\right]$ and $|\lambda(\omega, s)| \leq 1$. Define the functions

$$
\begin{aligned}
& c_{0}(\theta, \psi)=\int F(\theta, \psi, s)(1-s) d s, \\
& c_{1}(\theta, \psi, \omega)=\int F(\theta, \psi, s) \lambda(\omega, s) d s, \\
& c_{j}(\theta, \psi)=\int F(\theta, \psi, s) \zeta_{j}(s) d s \quad\left(1 \leq j \leq 2^{R}\right) .
\end{aligned}
$$

Substitution in $(* *)$ and integration in $\theta$ with respect to $\mu$ leads to the expression

(*) $n \int_{0}^{1} \int_{\Pi}\left\|c_{0}(\theta, \psi)+c_{1}(\theta, \psi, \omega)-\frac{1}{2} \sum_{r=0}^{R-1} \sum_{j=2^{r}+1}^{2^{r+1}} \chi_{j}(\omega) c_{j}(\theta, \psi)\right\|_{*(\psi)} d \omega \mu(d \theta)$.

The problem is reduced to minorating $(*)$. Notice that the contribution of the $c_{1}$-term in $(*)$ is bounded by $M^{2}$ and will therefore be negligible, provided the remainder has a minoration of order $\log n$.

3. Construction of an $H^{\infty}$-valued martingale. The idea of the proof is the fact that the terms appearing after the summation $\sum_{r=0}^{R-1}$ will sum up in the $l^{1}$-sense. To formalize this, we will exhibit a martingale in the variable $\omega$ such that pointwise a $c_{0}$-sequence in $H^{\infty}$ is obtained.

Define the function $W$ on $\Pi \times[0,1]$ by

$$
W(\theta, s)=\left|\bar{f}_{k}(\theta)\right| \text { for }(k-1) / n \leq s<k / n .
$$

Let $0<\varepsilon, \delta<1$ and $2>\rho>1$ be constants to be specified later. Define

$$
A_{j}(\theta, \psi)=\delta\left[\int \zeta_{j}+\varepsilon \int W(\theta, s) \zeta_{j}(s) d s\right]^{-1} \int F(\psi, \theta, s)_{\zeta_{j}}(s) d s
$$

which is an $\varepsilon^{-1} \delta M$-bounded $H^{\infty}$-function in $\psi$. Define for $r=0,1, \ldots, R-1$

$$
\Phi_{r}(\omega, \theta, \psi)=\sum_{j=2^{r}+1}^{2^{r+1}} \chi_{j}(\omega) A_{j}(\theta, \psi)
$$

and apply Lemma 3 to the function $\alpha(\psi)=\sum_{t=0}^{r-1} \rho^{r-t}\left|\phi_{t}(\omega, \theta, \psi)\right|$ for fixed $\omega, \theta$. This gives a function $\Psi_{r}(\omega, \theta, \psi)$ only dependent on the first $(r-1)$-levels of the diadic filtration. Therefore the sum

$$
\Gamma=\sum_{r=0}^{R-1} \Phi_{r} \cdot \Psi_{r} \quad\left(\Psi_{0}=1\right)
$$

is a diadic martingale difference sequence with respect to the Haar system in $\Omega$. Moreover, $\Gamma$ is $H^{\infty}$ in $\psi$ and since by construction

$$
\left|\Psi_{r}\right| \cdot\left|\Phi_{t}\right| \leq \rho^{-(r-t)} \text { for } r>t
$$

it follows from the lemma stated at the end of this section that

$$
\|\Gamma\|_{\infty} \leq C\left(\varepsilon^{-1} \delta M\right)(\rho-1)^{-1} \text { for } \delta \leq \varepsilon M^{-1} .
$$


Also (by (iii) of Lemma 3), freezing $\omega, \theta$ and considering the $L^{2}(\mu)$-norm in $\psi$, we have

$$
\left\|1-\Psi_{r}\right\|_{L^{2}(\mu)} \leq \sum_{t<r} \rho^{r-t}\left\|\Phi_{t}\right\|_{L^{2}(\mu)}
$$

Using $\Gamma_{\omega, \theta}$ as a test function, the following minoration of $(*)$ is obtained:

$$
\begin{array}{r}
n C^{-1} \varepsilon^{1 / 2} \delta^{-1 / 2} M^{-1 / 2} \int_{0}^{1} \int_{\Pi} \int_{\Pi}\left\{\sum_{r=0}^{R-1} \sum_{j=2^{r}+1}^{2^{r+1}} \chi_{j}(\omega)^{2} c_{j}(\theta, \psi)\right. \\
\left.A_{j}(\theta, \psi) \Psi_{r}(\omega, \theta, \psi)\right\} \omega(d \theta) \mu(d \psi) d \omega .
\end{array}
$$

The integral is minorated as

$$
\begin{aligned}
& \left|\sum_{0 \leq r<R}^{(* *)} 2^{-r} \sum_{j} \int_{\Pi} \int_{\Pi} c_{j}(\theta, \psi) A_{j}(\theta, \psi) \mu(d \theta) \mu(d \psi)\right| \\
& \quad-\varepsilon^{-1} \delta M \sum_{r=0}^{R-1} \int_{0}^{1} \int_{\Pi} \int_{\Pi}\left|\sum_{j} \chi_{j}(\omega)^{2} c_{j}(\theta, \psi)\right|\left|1-\psi_{r}(\omega, \theta, \psi)\right| \mu(d \theta) \mu(d \psi) d \omega .
\end{aligned}
$$
Then

LeMMA 4. Assume $0 \leq a_{k} \leq A, 0 \leq b_{k} \leq B$ and $a_{k} b_{k+j}<\rho^{-j}$ with $\rho>1$.

$$
\sum a_{k} b_{k} \leq 2 \rho A B / \rho-1
$$

We leave the proof as an exercise.

4. Contribution of main terms. It will be convenient to consider the function $d$ on $[0,1]^{2}$ defined by

$$
d\left(s, s^{\prime}\right)= \begin{cases}1 & \text { if } s, s^{\prime} \text { belong to the same interval }\left[\frac{k-1}{n}, \frac{k}{n}[\right. \\ 0 & \text { otherwise }\end{cases}
$$

It follows from the biorthogonality hypothesis that

$$
\int F(\theta, \psi, s) F\left(\psi, \theta, s^{\prime}\right) \mu(d \psi)=d\left(s, s^{\prime}\right) F(\theta, \theta, s) .
$$

Hence

$$
\begin{aligned}
\int_{\Pi} \int_{\Pi} c_{j}(\theta, \psi) A_{j}(\theta, \psi)= & \delta \int_{\Pi} \int_{0}^{1} \int_{0}^{1} d\left(s, s^{\prime}\right) \zeta_{j}(s) \zeta_{j}\left(s^{\prime}\right) F(\theta, \theta, s) \\
& \times\left[\int \zeta_{j}+\varepsilon \int W(\theta, s) \zeta_{j}(s) d s\right]^{-1} d s d s^{\prime} \mu(d \theta)
\end{aligned}
$$

where

$$
\left|[\cdots]^{-1}-\left(\int \zeta_{j}\right)^{-1}\right| \leq \varepsilon\left(\int \zeta_{j}\right)^{-2} \int W(\theta, s) \zeta_{j}(s) d s .
$$

This gives the contribution $C^{-1} \delta / n$ up to a perturbation of at most

$$
\frac{1}{n} \varepsilon \delta\left(\int \varsigma_{j}\right)^{-2}\left\{\int\|W(\theta, s)\|_{L^{2}(\mu) \zeta_{j}}(s) d s\right\}\left\{\int\|F(\theta, \theta, s)\|_{L^{2}(\mu) \zeta_{j}}(s) d s\right\}
$$


and thus $(C / n) \varepsilon \delta M^{3}$. Consequently, for $\varepsilon \sim M^{-3}$, the sum of the first integral in $(* *)$ has the contribution $\delta R / C n$.

5. Estimation of error terms. Again by Cauchy-Schwarz, the $r$ th triple integral appearing in $(* *)$ is dominated by

$$
\int_{0}^{1}\left\|\sum_{j=2^{r}+1}^{2^{r+1}} \chi_{j}(\omega)^{2} c_{j}(\theta, \psi)\right\|_{L^{2}(\mu(d \theta) \otimes \mu(d \psi))}\left\|1-\Psi_{r}(\omega, \theta, \psi)\right\|_{L^{2}} d \omega .
$$

For each $\omega \in[0,1]$, the first factor is dominated by

$$
\sup _{2^{r} \leq j \leq 2^{r+1}}\left\|c_{j}(\theta, \psi)\right\|_{L^{2}} \leq C M^{2} n^{-1 / 2} \sqrt{2}^{r} .
$$

Since for fixed $\omega, \theta$

$$
\left\|1-\psi_{r}\right\|_{L^{2}}(\mu(d \psi)) \leq \sum_{t<r} \rho^{r-t}\left\|\phi_{t}\right\|_{L^{2}(\mu(d \psi))}
$$

we find

$$
\int_{0}^{1}\left\|1-\Psi_{r}(\omega, \theta, \psi)\right\|_{L^{2}} d \omega \leq \sum_{t<r} \rho^{r-t} \sup _{2^{t}<j \leq 2^{t+1}}\left\|A_{j}\right\|_{L^{2}}
$$

where

$$
\left\|A_{j}\right\|_{L^{2}} \leq \delta\left(\int \zeta_{j}\right)^{-1}\left\|\int F(\psi, \theta, s) \zeta_{j}(s) d s\right\|_{L^{2}} \leq C \delta M^{2}\left(\int \zeta_{j}\right)^{-1}\left\|\zeta_{j}\right\|_{2} n^{-1 / 2}
$$

Thus

$$
\int_{0}^{1}\left\|1-\Psi_{r}\right\|_{L^{2}} d \omega \leq C\left(\sum_{t<r} \rho^{r-t} \sqrt{2}^{t}\right) \delta M^{2} n^{-1 / 2} \leq C \delta M^{2} n^{-1 / 2} \sqrt{2^{r}}
$$

choosing $2 \rho=1+\sqrt{2}$. Therefore, the second term in $(* *)$ is at most

$$
\frac{C}{n} \varepsilon^{-1} \delta^{2} M^{5} R=\frac{C}{n} M^{8} \delta^{2} R
$$

6. End of proof. The left member in the inequality stated in the Theorem dominates $(*)$ and hence, collecting previous estimates,

$$
C^{-1} M^{-4} \delta^{-1} n\left\{\frac{\delta R}{C n}-\frac{C}{n} M^{8} \delta^{2} R\right\}=C^{-1} M^{-4} R\left(1-C \delta M^{8}\right) .
$$

Taking $\delta \sim M^{-8}$, the Theorem follows with $\rho(M) \sim M^{-4}$.

Denote by $L_{n}^{\infty}\left(\right.$ resp. $\left.U_{n}\right)$ the linear space $\left[1, e^{i \theta}, \ldots, e^{i n \theta}\right]$ equipped with $L^{\infty}$ norm (resp. \|\|$_{U}$ ). As a consequence of the proof of the Theorem and a result of [4] we have

COROLlaRY 3. There exists $\kappa>0$ such that $d\left(L_{n}^{\infty}, U_{n}\right) \geq(\log n)^{\kappa}$, where $d$ is the Banach-Mazur distance of normed spaces.

One can give a more qualitative version of the Theorem (in particular, not involving a change of measure) and extend Szarek's results [12] to the spaces $A$ and $L^{1} / H_{0}^{1}$. Thus

PROPOSITION 4. Any normalized basis of $L^{1} / H_{0}^{1}$ has a subsequence which is equivalent to the usual $l^{1}$-unit vector basis. If $\left(g_{n}\right)$ is a normalized basis of $A$, then, 
for some increasing sequence $\left(n_{k}\right)$ of positive integers, the map $\sum c_{n} g_{n} \mapsto\left(c_{n_{k}}\right)_{k=1}^{\infty}$ takes $A$ onto $c_{0}$.

This answers affirmatively Proposition 4.10 of $[\mathbf{1 2}]$.

\section{REFERENCES}

1. J. Bourgain, New Banach space properties of the disc algebra and $H^{\infty}$, Acta Math. (to appear).

2. __ Extension of $H^{\infty}$-valued operators and bounded bianalytic functions, Mittag-Leffler report N6, preprint, 1983.

3. __ Quelques propriétés linéares de l'espace des séries de Fourier uniformément convergentes, C.R. Acad. Sci. Paris Sér. I 295 (1982), 623-625.

4. J. Bourgain and A. Pelczynski, in preparation.

5. S. V. Bochkarev, Logarithmic growth of arithmetic means of Lebesgue functions of bounded orthonormal systems, Soviet Math. Dokl. 16 (1975).

6. __ Existence of a basis in the space of functions in the disk and some properties of the Franklin system, Math. USSR-Sb. 24 (1974), 1-16.

7. S. Kaczmarz and H. Steinhaus, Theorie der Orhogonalreihen, Monografie Mat., Band 6, PWN, Warsaw, 1935, reprint, Chelsea, New York, 1951.

8. J. Lindenstrauss and L. Tzafriri, Classical Banach spaces. I, Springer-Verlag, Berlin and New York, 1977.

9. A. M. Olevskii, Fourier series with respect to general orthogonal systems, Springer-Verlag, Berlin and New York, 1975.

10. A. Pelczynski, Banach spaces of analytic functions and absolutely summing operators, CBMS Regional Conf. Ser. in Math., No. 30, Amer. Math. Soc., Providence, R.I., 1977.

11. S. Szarek, Nonexistence of Besselian basis in $C(S)$, J. Funct. Anal. 36 (1980).

12. __ Bases and biorthogonal systems in the spaces $C$ and $L^{1}$, Ark. Mat. 17 (1979), 255-271.

Department of Mathematics, Vrije Universiteit Brussel, Pleinlaan 2, F7, 1050 BRUSSELS, BELGIUM 